\title{
Quaderni
}

QUADERNI Communication, technologies, pouvoir

67 | Automne 2008

Jeu vidéo et discours

\section{Storytelling, la machine à fabriquer des histoires et à formater les esprits, Christian Salmon}

\section{Benjamin Berut}

\section{(2) OpenEdition}

1 Journals

\section{Édition électronique}

URL : http://journals.openedition.org/quaderni/248

DOI : 10.4000/quaderni.248

ISSN : 2105-2956

Éditeur

Les éditions de la Maison des sciences de l'Homme

\section{Édition imprimée}

Date de publication : 5 octobre 2008

Pagination : 129-132

\section{Référence électronique}

Benjamin Berut, «Storytelling, la machine à fabriquer des histoires et à formater les esprits, Christian Salmon », Quaderni [En ligne], 67 | Automne 2008, mis en ligne le 13 décembre 2012, consulté le 21 septembre 2020. URL : http://journals.openedition.org/quaderni/248; DOI : https://doi.org/10.4000/ quaderni.248 


\section{storytelling, \\ la machine à \\ fabriquer des \\ histoires et \\ à formater \\ les esprits}

\section{Christian Salmon}

Tout commence par deux exemples, celui d'un jeu vidéo servant à la formation des soldats de l'armée américaine, et celui d'une séance de lecture d'un conte pour enfants ayant pour but de resserrer les liens entre les directeurs d'une entreprise. Il était une fois le storytelling donc, un mot dont Salmon fait le symbole d'un nouveau totalitarisme qui s'impose par le récit aux esprits assujettis, celui du soldat ou de l'employé et, plus loin, du citoyen ou du consommateur. Le projet de l'auteur est donc intéressant au départ, il propose de mettre en avant l'influence de la méthode du storytelling qui traverse différents domaines de la société américaine et conduit à la « mise en fiction de la réalité » de celle-ci. Mais, le livre devient, au final, l'illustration de la difficulté de cerner ce que Sfez décrit comme « les figures structurantes des milieux dirigeants $»^{1}$, et plutôt que de décrire une idéologie dominante, Salmon se contente de construire un terme repoussoir.

Les domaines que traverse le storytelling sont au nombre de quatre selon l'auteur : le marketing, le management, l'armée et la politique. Dans le marketing, le storytelling conduit à présenter le produit comme ayant une histoire qui lui fait incarner certaines valeurs, le consommateur achetant le produit pour montrer son accord avec ces valeurs qu'il partage. Dans le management, le storytelling sert à fédérer les employés alors que le modèle de l'entreprise fordiste laisse la place à des petites entités qui doivent pouvoir s'adapter au plus vite aux évolutions économiques. Là s'arrête la part intéressante du livre, qui mériterait déjà d'être approfondie (notamment sur l'influence de cette méthode sur la décision des entreprises qui pratiquent le management des émotions qui appelle au changement permanent). 
Car, Salmon tente ensuite de nous convaincre d'une alliance entre le Pentagone et Hollywood, où les seconds produiraient des fictions tendant à légitimer les actions des premiers grâce au « $c a$ ractère prescriptif des fictions hollywoodiennes et [à] leur fonction de légitimation d'actes anticonstitutionnels ou tout simplement immoraux » (p. 168). Alliance dont la seule preuve serait des réunions que tiennent entre eux producteurs et militaires, et dont la partie émergée serait les jeux vidéo servant à la formation des soldats, à l'élaboration desquels collaborent des studios de production. Mais c'est finalement la partie sur le storytelling en politique qui est la moins crédible. George W. Bush et son administration y seraient les grands méchants cyniques, capables d'exploiter n'importe quelle histoire pour asservir l'esprit des citoyens américains, flanqués des armées de spin doctors ne pouvant concevoir la politique que par son esthétisation et son exploitation des émotions.

Mais au-delà des manques de chaque partie, la question qui se pose est celle du fil rouge qui permet au storytelling de traverser les genres pour, chaque fois, assujettir les esprits du consommateur, de l'employé, du militaire ou du citoyen. Pour Salmon, le storytelling n'a qu'une différence de nature avec «l'art du récit qui, depuis les origines, raconte en l'éclairant l'expérience de l'humanité » (p. 20). Une affirmation jamais démontrée qui se retrouve telle quelle à la fin du livre avec l'affirmation que « les nouveaux récits que nous propose le storytelling, à l'évidence, n'explorent pas les conditions d'une expérience possible, mais les modalités de son assujettissement. » (p. 199). Où est la différence, serait-on tenté de dire. Où est la dégradation des ancien- nes fonctions du récit que décrivent Barthes ou Deleuze ? L'un des premiers manques du livre, c'est son absence d'historique du phénomène qu'il tente de décrire. Salmon date l'apparition du storytelling dans les années 90, en se contredisant parfois, et en oubliant les spin doctors des années 60 , et sans non plus donné un lieu de naissance à cette pratique. Est-ce une méthode politique héritée de la présidence Reagan qui se développe dans le marketing ou le management, ou une technique de séminaires d'entreprise qui se retrouve dans la formation des militaires américains ?

Deuxième manque, Salmon ne pose pas la question de l'élaboration de ces histoires, celle du formateur, du publicitaire ou du politique. Impossible de compter les multiples exemples qu'accumule avec plus ou moins de réussite l'auteur, mais on ne trouve qu'une seule généalogie : celle de l'Ashley's story, tirée de la rencontre, durant la campagne présidentielle de 2004, d'une adolescente dont la mère avait été tuée le 11 septembre, et de George W. Bush. L'image de ce dernier la réconfortant avait été massivement exploitée dans le cadre de sa réélection, notamment auprès des indécis. Les storytells (on pourrait proposer ce néologisme pour éviter la répétition du mot histoires) naissent-ils donc de l'opportunité ? Sans doute. De la pratique ? Sans doute aussi. Salmon ne pose même pas la question.

Le troisième manque est celui de l'influence, car sans forcément passer par des analyses de réception, on ne peut pas passer sur les théories des usages et gratifications, du two-step flow communication ou même d'une conception herméneutique du récit. D'autant que Salmon ne présente que des exemples réussis plus ou moins 
acceptables : Nike parvient à redorer son image, Bush gagne les élections, les soldats commettent des crimes de guerre à cause des jeux vidéo, le conflit irakien est accepté par toute la population américaine...

Ces trois manques conduisent donc à une image très négative des États-Unis, un pays totalement sous l'influence des histoires élaborées par un petit groupe. Car, en ne posant pas la triple question de la dégradation des anciennes formes du récit, de l'élaboration de ces histoires et de leur influence, Salmon tombe dans le simplisme où le seul lien entre les différents domaines où l'on retrouve le storytelling c'est une éminence grise qui les élabore dans le but de garder la population assujettie. Il souligne ainsi les évolutions structurelles communes des entreprises et de l'armée et leur concordance avec l'arrivée du storytelling, mais ne va pas plus loin. La structure conduit-elle au storytelling ? Ou est-ce celui-ci qui influe sur la structure?

Sous l'emprise de cette éminence grise, c'est l'Amérique entière qui est asservie, qui ne peut plus penser par elle-même, contrôlée par un petit groupe qui, dans l'ombre, la nourrit de ses histoires qui peuplent le marketing, l'entreprise et la politique. Mais Salmon oublie le journalisme d'opposition au profit de Fox News, la vague contestatrice hollywoodienne au profit de 24 heures chrono. Et au final, il ne parle donc pas tant d'un nouveau concept que de sa vision des États-Unis, et des évolutions du pays sous les présidences Reagan, Bush père et fils. Et nous touchons là au grand paradoxe de ce livre, c'est que, privé de la rigueur des outils d'analyse, il se tourne lui-même vers le storytelling pour défendre ses idées. Salmon distribue donc les rôles : les méchants, les «gourous » comme il les appelle; les gentils, auteurs et journalistes tentant de dénoncer l'affaire; et les traîtres, comme Amanda Tucker du programme de lutte contre le travail des enfants recrutée par Nike en pleine polémique sur les usines de la firme en Asie. De même, Salmon joue sur les émotions, comme lorsqu'il décrit des réunions entre membres du Pentagone et producteurs d'Hollywood. Et surtout, tente de convaincre en entrecroisant les références ${ }^{2}$ en citant des exemples tirés de fictions ou de la réalité.

Le meilleur exemple de ce « storytelling », c'est son analyse de la politique américaine, où des Républicains sans scrupule manipulent avec cynisme les émotions de l'opinion, alors que les Démocrates, eux, naïfs, se refusent à utiliser ces méthodes pour parler à la raison des électeurs. Parce qu'il parle aux émotions, le storytelling est donc rangé parmi les méthodes d'esthétisation du politique et constitue une étape sur le chemin du totalitarisme. On n'est pas loin d'une théorie du complot où, loin des regards, se réunissent producteurs d'Hollywood et responsables du Pentagone; où un « responsable politique américain de haut niveau » avoue cyniquement qu'ils sont un « empire » qui crée sa "propre réalité » (p. 172). Storytelling n'est plus alors un concept, mais un fétiche agité à chaque occurrence et dont les dérivés s'accumulent (storytelling corporate, storytelling management, storytelling digital) sans aucune définition.

On est donc finalement déçu par ce livre, car le projet de départ était intéressant : décrire les façons dont les récits peuvent devenir totalitaires. 
Mais l'explication de Salmon est trop simple : la droite américaine a mis en place des récits ayant pour but d'assujettir la population. L'auteur insiste sur le jeu du récit avec les émotions, mais oublie que la pratique du récit se fait en trois étapes où l'émotion ne joue que sur la troisième : lire, interpréter et aimer ${ }^{3}$. De même, si le jeu du récit sur les émotions conduit à la suspension de l'incrédulité décrit par Coleridge, celle-ci ne dure qu'un temps et, même si le livre accumule les lieux de storytelling, le citoyen américain ne saurait être en permanence sous son influence, comme toujours endormi dans un monde imaginaire. De plus, Salmon oublie que l'acceptation d'un récit dépend de son ordonnancement, à tout moment la suspension de l'incrédulité peut se briser sous l'effet d'une invraisemblance. Il existe toujours un lieu de l'interprétation en dehors des récits qui se multiplient, un lieu d'enjeu sans doute puisque les récits peuvent aussi se confronter, mais là-dessus, comme nous l'avons déjà dit, Salmon laisse de côté les récits qui contredisent ceux qu'il dénonce.

Le livre laisse donc le lecteur sur sa faim, projet ambitieux il se contente d'une accumulation de données parfois contradictoires en ne proposant pour toute explication que l'image d'un « Pouvoir » fabriquant des récits si nombreux, uniformes et dispersés qu'ils projettent l'Amérique entière dans un état d'assujettissement qui ne peut conduire qu'au totalitarisme. Quant au terme storytelling, il perd toute profondeur pour ne devenir qu'une insulte puisque c'est forcément l'autre qui est dans l'exploitation des émotions, passant à côté de la définition de ce que peut être un récit totalitaire.
$\mathrm{N} \cdot \mathrm{O} \cdot \mathrm{T} \cdot \mathrm{E} \cdot \mathrm{S}$

1. L. Sfez, «Les figures structurantes des milieux dirigeants (1970/2002)», in Quaderni n ${ }^{\circ}$ 50/51, pp. 125-140.

2. Nous renvoyons ici à ce que Ricœur décrit comme la référence croisée du récit. P. Ricœur, Temps et récit, Le Point, coll Essais, 1983.

3. Nous renvoyons ici à l'analyse par Ricœur de Mimèsis III, et plus particulièrement des rapports entre narrativité et référence. P. Ricœur, Temps et récit 1, op. cit. pp. 146-155. 